\title{
Arbeitsmarktregulierung unter Beschuss. Die neue europäische Arbeitsmarktpolitik am Beispiel der Reformpolitik Frankreichs unter François Hollande
}

\begin{abstract}
Zusammenfassung
Durch die Krisenbearbeitung der Europäischen Union hat sich ein europäischer Interventionismus herausgebildet, der die Rahmenbedingungen für eine nationale Arbeitsmarktregulierung einschränkt. Während in der Vergangenheit vor allem jene Länder im wissenschaftlichen Fokus standen, welche die europäischen Hilfsprogramme EFSF und ESM in Anspruch nahmen und dadurch unter der Kontrolle der sogenannten Troika standen, blieb der Einfluss des europäischen Interventionismus auf jene Länder unterbelichtet, welche weit weniger stark von der Krise betroffen waren. Am Beispiel der französischen Arbeitsmarktreformen Loi Macron und Loi El Khomri untersucht der Artikel den Einfluss europäischer Interventionsinstrumente auf die nationale Arbeitsmarktregulierung. Im Fokus dieses Beitrages steht der Einfluss des europäischen Interventionismus auf die französische Arbeitsmarktregulierung, während die innenpolitischen Ausgangsbedingungen für die Durchsetzung der arbeitsmarktregulierenden Reformpakete nur angeschnitten werden können.
\end{abstract}

Schlagwörter: Euro-Krise, Frankreich, Europäische Union, Europäische Integration, Arbeitsmarktpolitik, Reformpolitik

\section{Labour market regulation under pressure. The Impact of European interventionism on French labour market policy}

\begin{abstract}
The European Union's crisis management has resulted in a European interventionism that restrains the EU Member States ability to regulate national labour markets. In the past, most scientific analysis focused on deficit countries under the control of the European Troika. This article uses the examples of the labour market reforms in France, "Loi Macron" and "Loi El Khomri", to examine the influence of European institutions on labour market regulations in a country less affected by the crisis.
\end{abstract}

Key words: Eurozone crisis, France, European Union, European Integration, Labour market policy, Reform policy (JEL: J08, J5, O52)

* Felix Syrovatka MA, Jg. 1988, Doktorand an der Eberhard-Karls-Universität Tübingen, Institut für Politikwissenschaften, D - 10823 Berlin. E-Mail: felix.syrovatka@ fu-berlin.de

** Artikel eingegangen: 24.01.2017, revidierte Fassung nach doppelt-blindem Begutachtungsverfahren akzeptiert: 08.12.2017

Für Hinweise und Anmerkungen danke ich den beiden anonymen GutachterInnen sowie Thorsten Schulten, Astrid Achterberg und Etienne Schneider. 


\section{Einleitung}

In der Wirtschaftskrise kam es zu starken Veränderungen im institutionellen Gefüge der Europäischen Union (EU). Vor allem im Bereich der Wirtschafts- und Währungsunion wurde seit 2010 bausteinartig ein wirtschaftspolitisches Kontrollsystem zur Überwachung nationaler Politiken etabliert. Über Verordnungen und zwischenstaatliche Verträge wurden Instrumente verankert, welche im Rahmen der wirtschaftspolitischen Koordinierung und der makroökonomischen Überwachung, die Einfluss- und Sanktionsmöglichkeiten europäischer Institutionen auf die nationale Reformpolitik ausgeweitet und Entscheidungsbefugnisse von der nationalen auf die europäische Ebene verlagerten. Vor allem im Bereich der Arbeitsmarktregulierung waren massive Kompetenzverschiebungen zugunsten der europäischen Ebene zu beobachten, so dass verschiedene BeobachterInnen von einem europäischen arbeitsmarktpolitischen Interventionismus ${ }^{1}$ sprechen (Müller \& Schulten, 2015). Dieser, so die hier formulierte These, verändert die Rahmenbedingungen für die nationale Arbeitsmarktregulierung und zielt auf die Einschränkung der Handlungsspielräume von Gewerkschaften und Arbeitnehmerorganisationen durch die Dezentralisierung von Lohnfindungsund Tarifvertragssystemen sowie einer Flexibilisierung von Arbeitnehmerrechten.

Während die Wirkung des Interventionismus am Beispiel der sogenannten Defizitlän$\operatorname{der}^{2}$ ausreichend untersucht wurde, blieb die Analyse jener Länder, welche nicht direkt der Kontrolle europäischer Institutionen unterstanden, relativ unterbelichtet. Im Folgenden soll daher am Beispiel der französischen Arbeitsmarktregulierung unter François Hollande gezeigt werden, dass die europäische Austeritätspolitik auch auf jene Länder disziplinierend wirkt, welche dem europäischen Zentrum zugeordnet und bisher von der Krise weniger stark betroffen waren. Im Fokus dieses Beitrags steht der Einfluss europäischer Institutionen auf die nationale Arbeitsmarktregulierung. Dazu werden 1.) die inhaltliche Stoßrichtung der europäischen Krisenpolitik dargestellt, darauf aufbauend wird 2.) der arbeitsmarktpolitische Interventionismus exemplarisch am Beispiel der länderspezifischen Empfehlungen (LSE) für Frankreich zwischen 2011 und 2016 charakterisiert, um 3.) den Einfluss der EU am Beispiel der beiden arbeitsmarktregulierenden Reformprojekte, dem Loi Macron und Loi El Khomri, abzubilden.

\section{Europäische Arbeitsmarkt- und Beschäftigungspolitik vor dem Hintergrund der Krisenbearbeitung}

Die europäische Arbeitsmarkt- und Beschäftigungspolitik unterlag bis zur Krise einem permanenten Wandel. Trotz verschiedener Initiativen, welche eine stärkere Verbindlichkeit einforderten, behielt die europäische Arbeitsmarkt- und Beschäftigungspolitik ihren symbolischen Charakter. Zwar gab es mit den beschäftigungspolitischen Leitlinien oder den Fle-

1 Im Folgenden soll von einem ,,arbeitsmarktpolitischen Interventionismus“ gesprochen werden, auch wenn der Artikel primär arbeitsmarktregulierende Reformpolitiken betrachtet. Jedoch umfasst der ,,arbeitsmarktpolitische Interventionismus“ auch Eingriffe in die aktive und passive Arbeitsmarktpolitik der EU-Mitgliedsländer.

2 Als Defizitländer werden jene Länder bezeichnet, welche auf Kredite der EU oder des IWF angewiesen sind oder waren und an Memoranden of Understandings gebunden sind/waren. 
xicurity-Grundsätzen Instrumente zur Harmonisierung europäischer Arbeitsmarktpolitiken, jedoch fehlten für eine Durchsetzung der empfohlenen Maßnahmen die nötigen Sanktionsmechanismen (Erne, 2015).

Dieser Zustand änderte sich in Folge der Eurokrise. Die Durchsetzung der sogenannten New Economic Governance umfasste eine Reihe von Maßnahmen und Regelungen, welche über verschiedene Projekte seit Anfang 2010 sukzessiv umgesetzt wurden und auf eine Koordinierung der europäischen Wirtschaftspolitiken zielten. Dem krisenkonstitutionalistischen Umbau war eine Narrativverschiebung auf europäischer Ebene vorgelagert, welche ganz grundlegend von den europäischen Institutionen initiiert und moderiert wurde (Heinrich, 2015). Krisenursachen und -interpretation waren noch zu Beginn der Krise ebenso umkämpft wie mögliche Schritte zur Krisenlösung. Dabei standen sich bis Frühjahr 2010 südeuropäische und nordeuropäische Länder in einer hoch polarisierten Debatte gegenüber.

Die südeuropäischen Staaten unter Führung Frankreichs verorteten die wesentlichen Krisenursachen in den asymmetrischen Strukturen der Europäischen Währungsunion sowie in den Leistungsbilanzüberschüssen der exportorientierten Euro-Länder und plädierten für eine europäische Wirtschaftsregierung, die Einführung von Eurobonds und einen kollektiven fiskalpolitischen Transfermechanismus (Dyson, 2010). Die nordeuropäischen Mitgliedsstaaten sahen dagegen die hohe nationalstaatliche Verschuldung im Euroraum als wesentliche Krisenursache. Sie traten für eine Verschärfung der austeritätspolitischen Kriterien sowie für einen stabilitätsorientierten Kurs der Europäischen Zentralbank (EZB) ein. Die Einführung von kollektiven fiskalpolitischen Transfermechanismen oder einer gemeinsamen Wirtschaftsregierung wurde strikt abgelehnt und durch die Berufung auf die No-Bail-Out-Klausel gezielt verhindert (Schmidt, 2013). Je mehr die Krise sich zuspitzte, umso konfrontativer standen sich die unterschiedlichen und miteinander konkurrierenden Krisennarrative gegenüber, auch weil sich im Konflikt selbst die unterschiedlichen Akkumulationsregime und Regulationsweisen sowie die Gläubiger-Schuldner-Beziehung in der EU widerspiegelte (Overbeek, 2012).

Im Zuge der europäischen Krisenkoordinierung durch die Institutionalisierung der gesamteuropäischen Hilfsprogramme EFSF und ESM begannen die Europäische Kommission und die EZB moderierend in die Konfliktdynamik zwischen den großen EU-Mitgliedsstaaten einzugreifen. Mithilfe einer problemorientierten und technokratischen Form der Artikulation wurden die verschiedenen und miteinander konkurrierenden Krisendeutungen durch Initiativen der europäischen Institutionen in ein einheitliches wettbewerbsorientiertes Denkmuster überführt (Heinrich \& Jessop, 2015). Durch den gezielten Bezug auf bereits institutionalisierte EU-Strukturen, europäische Richtlinien und Übereinkommen belebte v.a. die Europäische Kommission den bereits in der Lissabon-Strategie formulierten Wettbewerbsdiskurs wieder und intensivierte diesen innerhalb eines neugeschaffenen europäischen Elitenkonsenses (Schmidt, 2013). Die Krise wurde als Wettbewerbskrise gerahmt und ihre Überwindung diskursiv an die Überwindung von Wettbewerbsunterschieden gekoppelt. Die Rückkehr zu fiskalischer Disziplin ebenso wie weitreichende Überwachung nationaler Wirtschaftspolitiken waren zu Eckpfeiler einer zukünftigen europäischen Wachstumsstrategie erklärt worden, was zugleich alternative Ansätze zur Krisenlösung delegitimierte. Damit folgten die europäischen Institutionen der Kriseninterpretation der nordeuropäischen Staaten und verteilten die Lasten für die Überwindung der Wettbewerbsungleichgewichte einseitig auf die südeuropäischen Staaten. Dennoch gelang es durch die Reproduk- 
tion wettbewerbsorientierter Vorstellungswelten und Kompromisskonstellationen, Frankreich und die südeuropäischen Staaten in den Elitenkonsens hegemonial miteinzubinden und damit die Spannungen zwischen den Mitgliedsstaaten zu entschärfen (Heinrich, 2015). Für die konsensuale Einbindung Frankreichs in das marktliberal-wettbewerbsorientierte Krisennarrativ war neben dem strategischen Agieren der europäischen Institutionen die strukturelle ökonomische und politische Schwäche des Landes während der Eurokrise zentral. Seit 2010 zeichnete sich in Frankreich eine schwere Rezession ab, welche mit einem Anstieg von Arbeitslosigkeit und Staatsverschuldung einherging (Heine \& Sablowski, 2015). In Verbindung mit den politischen Krisendynamiken wurde die Verhandlungsposition Frankreichs auf europäischer Ebene geschwächt, was zu einer Defensivposition gegenüber den nordeuropäischen Ländern führte. Diese Position, v.a. gegenüber Deutschland, wurde durch das seit 2009 laufende EU-Defizitverfahren sowie die mehrfache Herabstufung der Kreditwürdigkeit durch internationale Ratingagenturen weiter verstärkt. Es kam zu einer Asymmetrie im deutsch-französischen Bilateralismus (Schneider \& Syrovatka, 2017).

Mit der Durchsetzung des hegemonialen Krisennarrativs und der Verengung des Analysefokus auf die (preisliche) Wettbewerbsfähigkeit gelang es, das politische Kampffeld von den vorangegangenen Krisenphasen zu verschieben, alternative Politikansätze zu desartikulieren und die nationalen Arbeitsmarktregulierungs- und Lohnpolitiken in den Fokus der Krisenbearbeitung zu rücken. Daher basiert die EU-Krisenpolitik auf der Annahme, dass durch eine stärkere europäische Konvergenz in der Arbeitsmarktregulierung die Krisenursachen behoben werden können (Degryse, Jepsen \& Pochet, 2013). Die narrative Verknüpfung von nationaler Arbeitsmarktregulierung und europäischer Wettbewerbsfähigkeit begründete die Ausrichtung der europäischen Austeritätspolitik, welche auf der Grundlage schon bestehender arbeitsmarkt- und beschäftigungspolitischer Verfahren und Leitbilder ein umfassendes System zur Kontrolle nationaler Arbeitsmarktregulierung und Lohnpolitik etablierte (van Gyes \& Schulten, 2015).

$\mathrm{Zu}$ den wichtigsten Bausteinen der New Economic Governance gehört die Europa2020-Strategie, welche 2010 als Nachfolge der Lissabon-Strategie verabschiedet wurde. Diese reformulierte nicht nur die Flexicurity-Grundsätze im Zuge der Krisenbearbeitung neu, sondern etablierte mit dem Europäischen Semester einen umfassenden Politikzyklus zur Koordinierung der europäischen Wirtschaftspolitik. Ähnlich wie beim LuxemburgerProzess von 1997 stehen am Ende eines jeden Semesterzyklus politische Empfehlungen, deren Umsetzung und Wirksamkeit von der Europäischen Kommission überprüft werden. Im Fokus der Überwachung stehen sowohl die nationalen Haushalts-, wie auch die Struktur- und Makropolitiken, womit nicht nur die wirtschafts- und finanzpolitischen, sondern auch die nationalen arbeitsmarkt- und sozialpolitischen Strategien sowie die jeweilige Lohnentwicklung von der europäischen Überwachung betroffen sind (Rödl, 2012).

2011 und 2013 wurde das Europäische Semester durch zwei Gesetzespakete weiter ausgebaut und in seiner beschäftigungspolitischen Schwerpunktsetzung gestärkt. Die Erweiterung des Europäischen Semesters führte ein System der haushalts- und makropolitischen Überwachung ein, welches auf der Grundlage zuvor festgelegter Indikatoren (sog. Scoreboard) frühzeitige „makroökonomische Ungleichgewichte“ erkennen und beheben soll. Verknüpft ist dieses System mit finanziellen Sanktionen, welche verhängt werden können, wenn die von der Kommission formulierten politischen Empfehlungen auf Grund- 
lage des Warnmechanismus von den betroffenen Mitgliedsländern nicht erfüllt werden. Damit erlangt auch die Europäische Beschäftigungspolitik eine neue Stufe der Verbindlichkeit, da mit den Sanktionsmechanismen der rein freiwillige Charakter der politischen Empfehlungen verlassen wird. Zugleich rückt die europäische Lohn- und Arbeitsmarktpolitik ins Zentrum makroökonomischer Koordinierung, was sich nicht zuletzt in den makroökonomischen Zielmarken des Scoreboards niederschlägt, welche explizit auch Lohnstückkosten beinhalten (Oberndorfer, 2014).

\section{Herausbildung eines arbeitsmarkt- und lohnpolitischen Interventionismus}

Mit der Durchsetzung krisenkonstitutionalistischer Maßnahmen wurden Möglichkeiten für die Europäische Institutionen geschaffen, nicht nur direkten Einfluss auf die konkrete Ausgestaltung von Reformen im Bereich der Arbeitsmarktregulierung und Lohnpolitik zu nehmen, sondern zudem - bspw. über die LSE - Reformen anzustoßen, bzw. diese vom betroffenen Mitgliedsland zu verlangen (Syrovatka, 2016). In Anknüpfung an den Begriff von Schulten \& Müller (2013) wird die neue Qualität des Einflusses auf die nationale Arbeitsmarktregulierungs- und Lohnpolitik als europäischer, arbeitsmarktpolitischer Interventionismus gefasst. Die Interventionsformen lassen sich dabei nach ihrer spezifischen Qualität und Reichweite unterscheiden:

1. Die schwächste Form des europäischen Interventionismus zeigt sich in den europäischen Politikformulierungen im Rahmen unterschiedlicher Konvergenzinitativen (u.a. beschäftigungspolitische Leitlinien) sowie den LSE des Europäischen Semesters. Die politischen Empfehlungen haben durch die Einführung finanzieller Sanktionen einen verbindlicheren Charakter bekommen, auch wenn bisher keine Strafzahlungen von Seiten der Kommission verhängt wurden ${ }^{3}$. Der Druck, die politischen Empfehlungen umzusetzen, beruht hier primär in der impliziten Androhung von Strafzahlungen oder in der Vorlage eines Korrekturmaßnahmeplans.

2. Die mittlere Form des Interventionismus umfasst die Krisenbearbeitung durch die EZB. Im Gegenzug zum Ankauf von Staatsanleihen verlangt die EZB politische Reformen, u.a. im Bereich der Arbeitsmarkt- und Sozialpolitik. Wurde diese Politik zu Krisenbeginn noch informell betrieben (etwa in Italien 2011), ist sie seit 2012 offizielle Zentralbankpolitik.

3. Die stärkste Form des Interventionismus zeigt sich in jenen Ländern, welche sich im Rahmen sog. „Memoranden of Understandings“ zu weitreichenden Arbeitsmarktreformen gegenüber der Troika, bzw. den Institutionen aus IWF, EZB, Europäischer Kommission und ESF, im Tausch für finanzielle Hilfen, verpflichtet haben (Rödl \& Callsen, 2015). Diese Interventionsform ermöglicht eine direkte Einflussnahme der europäischen Institutionen auf die nationale Arbeitsmarktpolitik, ist die Umsetzung der politischen Forderungen doch Voraussetzung für die Auszahlung von Krediten. Der Spiel-

3 Die jüngsten Beispiele, Portugal und Spanien, zeigen, dass zwar keine Strafzahlungen verhängt, jedoch die EU-Strukturmittel für 2017 eingefroren wurden. 
raum für eine eigenständige Arbeitsmarktpolitik ist damit vollständig verstellt, da sämtliche arbeitsmarktregulierende Maßnahmen mit der Troika, bzw. den Institutionen, abgestimmt werden müssen.

Der Fokus aller Interventionsformen liegt auf einer Flexibilisierung der Löhne als zentraler makroökonomischer Anpassungsfaktor. Da unmittelbare politische Steuerungsmöglichkeiten oftmals nur im Bereich des öffentlichen Dienstes sowie mit Bezug auf das Niveau gesetzlicher Mindestlöhne existieren, kann eine Flexibilisierung der Löhne nur über eine Deregulierung des Lohnfindungsumfelds, einer Dezentralisierung und Verbetrieblichung der Lohnfindung sowie einer Schwächung der Gewerkschaften erreicht werden. Diese Stoßrichtung wird in einem zentralen Dokument der Kommission konkretisiert. Mit dem Ziel, eine „business-friendly environment“ (COM, 2012, S. 75) zu schaffen, entwickelt die Kommission zahlreiche arbeitsmarktregulierende Vorschläge für „beschäftigungsfreundliche Reformen“ (COM, 2012, S. 71), welche u.a. die Reduzierung des Kündigungsschutzes, die Anhebung des Rentenalters, Senkung der Arbeitslosenhilfe, Senkung des Mindestlohnes, Reduzierung der Tarifbindung und die Dezentralisierung des Tarifvertragssystems beinhalten. Mittelfristig sollen diese Maßnahmen zu einer „overall reduction in the wagesetting power of trade union“ (COM, 2012, S. 104) führen. Ein Blick auf die arbeitsmarktpolitischen Reformen in den Defizitländern macht deutlich, dass diese Vorschläge die inhaltliche-strategische Klammer für die arbeitsmarktpolitischen Reformbestrebungen des neuen europäischen Interventionismus darstellen (Müller, Schulten \& Zuckerstätter, 2015).

Damit umfasst der europäische Interventionismus nicht nur eine tendenzielle Beschneidung nationalstaatlicher Souveränität und eine Kompetenzverlagerung von der nationalen auf die europäische Ebene, sondern hat zugleich massive Einschränkungen gewerkschaftlicher Handlungsspielräume zur Folge (Müller \& Platzer, 2016). Dieser Umstand beschränkt sich nicht nur auf die Defizitländer, sondern umfasst ebenso - in abgemilderter Form - alle europäischen Mitgliedsstaaten.

\section{Französische Arbeitsmarktregulierung unter Druck}

Im April 2009 leitete die Europäische Kommission ein Defizitverfahren gegen Frankreich ein. In Folge von Konjunkturpaketen und Bankenrettung waren die französischen Staatsschulden im Krisenjahr 2008 deutlich stärker gestiegen als im Jahr zuvor (Jabko \& Massoc, 2012). Aufgrund der europaweiten Krisenprozesse wurde das Defizitverfahren jedoch auf fiskalpolitische Empfehlungen beschränkt, die eine langfristige Reduzierung des Haushaltsdefizits auf 2,9\% bis 2012 vorsahen. Dennoch geriet mit der Einleitung des Defizitverfahrens die französische Arbeitsmarktregulierung verstärkt in den Fokus der Kommission. Vor allem in Folge des 2013 eingeleiteten Verfahrens bei makroökonomischen Ungleichgewichten unterlag die französische Arbeitsmarktregulierung einem speziellen Monitoringverfahren.

Ganz im Sinne des hegemonialen Krisennarrativs stellte der Europäische Rat in seinen LSE von 2011 bis 2017 durchgehend fest, dass Frankreich neben einer Schuldenkrise auch an einer Wettbewerbskrise und einer strukturellen Arbeitsmarktschwäche leide. Die Wettbewerbskrise ergibt sich für den Europäischen Rat aus der preislichen und nicht-preislichen 
Wettbewerbsschwäche infolge zu hoher Arbeitskosten und geringer Unternehmensflexibilität in ,,allen Aspekten der Beschäftigungsbedingungen“ (ER, 2016, S. C299/116). Primär der Mindestlohn und seine spezifische Indexierungsform sowie die „hohe Belastung“ der Arbeitgeber durch Steuern und Sozialabgaben gelten der EU als Ursache. Die strukturelle Schwäche des Arbeitsmarktes resultiert dagegen für die EU-Institutionen aus der starken Segregation, dessen Ursachen wiederum in den zu starren Kündigungsschutzvorschriften sowie in den fehlenden Ressourcen der Arbeitsämter und einer zu wenig aktivierenden Arbeitslosenpolitik liegen. Die Konstanz, mit der die europäischen Institutionen die französische Krise analysieren, spiegelt sich auch in den ,empfohlenen“ Maßnahmen wider. In allen LSE zwischen 2011 und 2017 wird Frankreich aufgefordert, die Mindestlohnentwicklung zu drosseln, reglementierte Gewerbe und Berufe zu liberalisieren sowie Kündigungsschutzvorschriften zu lockern.

Trotz der Kontinuität in der Ursachenanalyse wandelte sich der Fokus der LSE in Folge zunehmender Krisenverschärfung. Seitdem Frankreich ab 2013 dem Verfahren makroökonomischer Ungleichgewichte und damit einem speziellen Monitoring unterlag, rückten die arbeitsmarktregulierenden Arrangements in den Mittelpunkt der LSE. Während vor 2013 die Empfehlungen für die Arbeitsmarktregulierung eher allgemein gehalten waren und hauptsächlich die hohen Arbeitskosten als Ursache für die (preisliche) Wettbewerbsschwäche bemängelten, können ab 2013 eine deutlich detailliertere arbeitsmarktpolitische Analyse und Empfehlungen konstatiert werden.

Trotz der nun auch nicht-preislichen Wettbewerbsfaktoren umfassenden Analyse blieben die empfohlenen Maßnahmen relativ allgemein und waren wenig spezifisch, lag doch der Schwerpunkt der LSE auf arbeitsmarkt- und lohnpolitischen Maßnahmen. Vor allem die Lohnfindung sowie arbeitsgesetzliche Regelungen, wie die 35-Stunden-Woche, gerieten in den Fokus der europäischen Wettbewerbsanalyse. So schrieb der Rat (ER 2014) in die LSE für Frankreich: ,Wage-setting in France tends to result in distortion of the wage structure and limit the ability to firms to adjust wages in economic downturns“" (S. C247/45). Die spezifische Form der Lohnfindung und seine Kopplung an die Entwicklung des Mindestlohnes würden der Produktivitätsentwicklung entgegenstehen sowie zu einer Verringerung der Wettbewerbsfähigkeit und der Gewinnspanne der Unternehmen führen (ER, 2015a). Trotz des Auroux-Gesetzes von 1982 wurden Tarifvereinbarungen in Frankreich weiterhin auf der Branchenebene beschlosssen, auch wenn seitdem Ausnahmen möglich waren. Daher empfahl der Europäische Rat mit Nachdruck eine Reform und Dezentralisierung des Lohnbildungssystems, ,to ensure that wages evolve inline with productivity“ (ER, 2015a, S. C272/55) sowie die Schaffung weiterer Abweichmöglichkeiten zu Branchentarifverträgen. Darüber hinaus kritisierte er die arbeitsmarktpolitischen Arrangements ebenso wie den Kündigungsschutz als zu starr, was die Unternehmensflexibilität auf wirtschaftliche Schwankungen zu reagieren massiv einschränken würde. Vor allem die Arbeitszeitregelungen seien grundlegend verantwortlich für die geringe Wettbewerbsfähigkeit der Unternehmen. Daher empfahl der Rat (2015a) eine Flexibilisierung der Arbeitszeiten und eine weitere Lockerung des Kündigungsschutzes:

\footnotetext{
"Reform the labour law to provide more inentives for employers to hire an open-ended contract. Facilitate take of derogations at company and branch level from general legal provisions, in particular as regards working time arrangements" (S. C 272 \& 55).
} 
Zugleich verbanden die europäischen Institutionen ihre arbeitsmarktregulierenden Empfehlungen mit der konkreten Androhung von Strafzahlungen. So stellte die Kommission in einem Bericht aus dem Mai 2013 fest, dass die nachfrageorientierten Maßnahmen der neugewählten französischen Regierung Ayrault den LSE widersprächen. Die Kommission erhöhte daraufhin den Druck, knüpfte eine Fristverlängerung für den Abbau der Haushaltsdefizite an eine schärfere Überwachung und drohte mit der Verhängung von Strafzahlungen. Frankreich musste im Oktober 2013 einen Aktionsplan sowie ein „,haushaltspolitisches Partnerschaftsprogramm“ vorlegen und gegenüber der Europäischen Kommission und dem Europäischen Rat die geplanten Strukturmaßnahmen erläutern (Syrovatka, 2016). Nach Ablauf der Fristverlängerung wiederholte sich die Prozedur erneut und die Kommission drohte im Februar 2015 in einer offiziellen Vorlage für den Europäischen Rat mit der Verhängung eines Korrekturmaßnahmeplans und finanziellen Sanktionen (COM, 2015). In der vom Rat beschlossenen Vorlage wird von diesem Schritt abgesehen, jedoch eine sechsmonatige Frist vereinbart, in der konkrete Maßnahmen umgesetzt und das Land sich einem erneuten Monitoring zu unterziehen hat (ER, 2015b; GDECFIN, 2015). Zugleich wurde eine stärkere halbjährliche Überwachung der Reformschritte primär im Bereich der Arbeitsmarktregulierung durch die Kommission vereinbart (ER, 2015b; COM, 2016).

Neben den europäischen Institutionen äußerten sich auch Regierungspolitiker anderer EU-Mitgliedsstaaten zur arbeitsmarktpolitischen Situation Frankreichs. Vor allem Mitglieder der deutschen Bundesregierung sowie PolitikerInnen der Regierungsfraktionen sprachen sich öffentlich für eine tiefgreifende Reform der französischen Arbeitsmarktregulierung aus (Lux, 2016). Der deutsche Finanzminister Wolfgang Schäuble kritisierte im April 2015 die geringen französischen Reformfortschritte in der Arbeitsmarktregulierung und regte an, dass das französische Parlament, ähnlich wie in Spanien, doch mit Zwang von der „Notwendigkeit der Arbeitsmarktreform überzeugt“" ${ }^{\text {"W }}$ werden könnte. Zudem wurde mehrmals von Seiten der deutschen Bundesregierung darauf hingewiesen, dass die Bundesrepublik die Zustimmung zu einer Verlängerung des Defizitverfahrens im Europäischen Rat verweigern könne, sollte die französische Regierung keine überzeugenden reformpolitischen Schritte in der Arbeitsmarktregulierung einleiten (Clift \& Ryner, 2014).

Insgesamt lässt sich beobachten, dass der Druck auf Frankreich, arbeitsmarktregulierende Reformen durchzusetzen, seit der Wahl von François Hollande zum französischen Präsidenten mit dem Europäischen Semester 2013 stark zugenommen hatte. Frankreich wurde für seine Arbeitsmarktregulierung von verschiedenen Seiten kritisiert und bekam mehrfach finanzielle Sanktionen angedroht.

Zugleich zeigt sich, dass die getrennten Instrumente des europäischen Semesters und des Defizitverfahrens miteinander verschmelzen. Im Folgenden soll der Einfluss der europäischen Institutionen und ihrer politischen Empfehlungen am Beispiel der Umsetzung und inhaltlichen Ausgestaltung zweier zentraler arbeitsmarktregulierender Reformprojekte dargestellt werden.

Um das Zusammenspiel zwischen nationaler und europäischer Ebene im Folgenden analytisch fassen zu können, wird die EU im Anschluss an die staatstheoretischen Überle- 
gungen von Nicos Poulantzas (2002) als „Verdichtung 2. Ordnung“5 (Brand et al., 2007, S. 228) verstanden. Damit wird auf die zentrale Stellung der Nationalstaaten in der europäischen Politikformulierung verwiesen und den europäischen Institutionen eine eigene Dichte und eine relative Eigenständigkeit zugestanden (Bieling \& Brand, 2015). Eine solche analytische Perspektive auf die EU lässt zugleich die nationalen Kräfteverhältnisse gegenüber europäischen Politiken als eine Art Filter verstehen. Je nach spezifischer Konfiguration der nationalen Kräfteverhältnisse ergibt sich in den europäischen Kernstaaten ${ }^{6}$ die Qualität und Reichweite europäischer Entscheidungen und Politikempfehlungen, da die Stoßrichtung auf nationaler Ebene entweder abgeschwächt, gedämpft oder verstärkt wird.

\section{5. "Zeichen an unsere europäischen Partner" - Das Loi Macron}

Mit dem Loi pour la croissance, l'activité et l'égalité des chances économiques stellte die Regierung von Manuel Valls im November 2014 ihr erstes umfassendes Arbeitsmarktpaket ${ }^{7}$ vor. Waren zuvor mit dem Pacté de responsabilité und der Einführung des sogenannten CICE-Programms die Unternehmen steuerlich und abgabenmäßig erheblich entlastet worden, so beinhaltete das Loi Macron nun eine ganze Reihe von explizit arbeitsmarktregulierenden Maßnahmen und Eingriffen in die Arbeitsbeziehungen.

Die neue arbeitsmarktpolitische Agenda war dabei kontextuell in eine angebotspolitische Wende der Regierung eingebettet ${ }^{8}$. Nach der Niederlage der Sozialistischen Partei (PS) bei den Kommunalwahlen und dem Verlust der Senatsmehrheit im Frühjahr 2014 hatte Hollande im April und im August zweimal sein Kabinett umgebildet. Während Kritiker einer angebotspolitischen Wirtschaftspolitik aus der Regierung entfernt wurden, stärkte die Kabinettsumbildung den rechten, unternehmerfreundlichen Parteiflügel. Mit Manuel Valls (Ministerpräsident) und Emmanuel Macron (Wirtschaftsminister) wurden zwei Vertreter einer marktliberalen Wirtschaftspolitik in Schlüsselpositionen der Regierung berufen (Méda, 2017).

Die Kabinettsumbildung und die damit einhergehende wirtschaftspolitische Wende der Regierung resultierten zum einen aus dem innen- wie außenpolitischen Scheitern der ne-

5 Die supranationale Staatlichkeit der EU vermittelt sich aus dieser Analyseperspektive zum einen über nationalstaatliche Apparate, die nach wie vor als Verdichtung von Interessen von Klassen und Klassenfraktion 1. Ordnung gefasst werden, und zum anderen über transnationale Kommunikations- und Koordinationsarenen (Bieling \& Hüttmann, 2016).

6 Die Position innerhalb der europäischen Arbeitsteilung ist neben der spezifischen Konfiguration des nationalen Kräfteverhältnisses von zentraler Bedeutung für die Reichweite und Qualität des europäischen Interventionismus (Simonazii, Ginzburg \& Nocella, 2013).

7 Im Gesetzgebungsprozess wurden dem Reformpaket auch nicht den Arbeitsmarkt betreffende Regelungen hinzugefügt. So beinhaltet das verabschiedete Gesetz u.a. Regelungen zur Endlagerung radioaktiver Abfälle. Im Folgenden werden nur die arbeitsmarktpolitisch relevanten Maßnahmen näher betrachtet.

8 Die Umbildung der Regierung und die Ernennung von Emmanuel Macron muss als Start- und Wendepunkt für eine neue Form der Reformpolitik verstanden werden. Im Unterschied zum 2013 verabschiedeten ,Loi de sécurisation de l'emploi“ beruhen die nachfolgenden Reformen in der Arbeitsmarktregulierung nicht mehr auf den Konsultationen mit den Konfliktpartnern (Bazin, 2015). 
okeynsianischen Politik während der ersten Phase der Amtszeit Hollandes. Seine im Wahlkampf angekündigten Reformen - wie die Regulierung des Banken- und Finanzsektors wurden verwässert oder gar nicht erst angegangen. Zugleich blieb die französische Regierung auf europäischer Ebene in ihrer Defensivposition verhaftet.

Zum anderen etablierte sich auf der nationalen Ebene das wettbewerbsorientierte Krisennarrativ der EU. Vermittelt über den vielbeachteten „Gallois-Report“ des ehemaligen Airbus-Vorsitzenden Louis Gallois, einer Kampagne des größten französischen Unternehmensverbandes MEDEF und den Empfehlungen der Europäischen Kommission, wurde die Krise als Wettbewerbskrise neu gerahmt. Vor dem Hintergrund wachsender Arbeitslosigkeit und fehlender ökonomischer Dynamik konnte sich jene Erzählung durchsetzen, welche die Ursachen für die französische Wirtschaftskrise in der Regulation des Arbeitsmarktes und den im europäischen Vergleich relativ hohen Lohnstückkosten verortete (Lemoine, 2016; exemplarisch: Aghion, Cette \& Cohen, 2014).

Die schnelle Erholung der deutschen Wirtschaft nach dem Kriseneinbruch ließen zudem die Arbeitsmarktreformen der Agenda 2010 zum Leitbild für die Erneuerung des französischen Arbeitsmarktes werden (Duval, 2013; Neumann, 2017). Vor allem MEDEF bezog sich positiv auf die deutschen Reformen. In der groß angelegten Kampagne „France2020“, welche in Anlehnung an die deutsche Agenda 2010 eine Zukunftsvision für ein „wettbewerbsfähiges Frankreich“ im Jahr 2020 zeichnete, betonte MEDEF die Notwendigkeit wettbewerbsfähiger Unternehmen für die Überwindung von Arbeitslosigkeit und Wachstumskrise. Kern der Argumentation war die grundlegende Umstrukturierung des französischen Arbeitsmarktes durch eine umfangreiche Deregulierung und Flexibilisierung (MEDEF, 2014b).

In drei Positionspapieren listete der Arbeitgeberverband zahlreiche Reformvorschläge auf. So finden sich in den Positionspapieren u.a. die Forderung nach einer Reform des Arbeitsrechts im Hinblick auf Flexibilisierung von Arbeitszeiten und Kündigungsschutz und die Forderung nach einer Stärkung der Unternehmensebene für Vereinbarungen zwischen den Konfliktpartnern sowie zur Schaffung von Ausnahmen zum Mindestlohn (MEDEF, 2014a; 2014b). Mit diesen teils sehr konkreten Reformvorschlägen intervenierte MEDEF in die öffentliche Debatte zur Zukunft der französischen Wirtschaft und setzte die Regierung unter erheblichen Handlungsdruck.

Diese reagierte auf die Kampagne des Unternehmensverbandes, indem der neu berufene Wirtschaftsminister Emmanuel Macron im Vorfeld des Gesetzgebungsprozesses verstärkt den Dialog mit den Unternehmensverbänden suchte ${ }^{9}$. Zugleich näherte sich Macron der Argumentation von MEDEF an und analysierte mit la défiance, la complexité et le corporatisme drei zentrale Krankheiten der französischen Wirtschaft, welche es durch strukturelle Reformen zu überwinden galt ${ }^{10}$. Ausgehend von dieser Analyse zielte die inhaltliche Ausgestaltung des Reformpakets primär auf die preisliche Wettbewerbsfähigkeit der französischen Wirtschaft und kongruierte mit zahlreichen politischen Empfehlungen der europäischen Institutionen. Im Vordergrund des Reformpaketes stand die Liberalisierung von bisher beschränkten Berufen, die Schaffung von Rechtssicherheit für Arbeitgeber bei Kündigungen und Massenentlassungen sowie die Flexibilisierung von Arbeitnehmerrechten,

$9 \quad$ Le Monde, 31.08.2014

10 Le Monde, 16.10.2014 
insbesondere des Kündigungsschutzes (Sterdyniak, 2015). Konkret beinhaltete das Gesetzespaket u.a.:

- einen massiven Abbau von Zugangsbeschränkungen, wie Numerus Clausus oder anderer Barrieren für bisher regulierte Berufe, wie Notare oder Taxisfahrer.

- die Ausweitung der Sonntags- und Nachtarbeit: Zulassungserweiterung für Geschäfte, die sonntags oder bis Mitternacht geöffnet haben dürfen. Zugleich wurde die Möglichkeit geschaffen, auf Grundlage von Betriebsvereinbarungen Nacht- und Sonntagsarbeit auch in Gebieten anzuordnen, die nicht als Tourismusgebiete klassifiziert sind.

- die Verlängerung von sog. „Arbeitsplatzsicherungsvereinbarungen“ von zwei auf fünf Jahre. Die sog. „Arbeitsplatzsicherungsvereinbarungen“ sind betriebliche Vereinbarungen zwischen den Gewerkschaften und den Arbeitgebern und sehen auf eine begrenzte Zeit Abweichungen bei den allgemeinen Bestimmungen über Arbeitszeitdauer, ihre Verteilung sowie der Vergütung für die Arbeitnehmer im Gegenzug zur eigenen Arbeitsplatzsicherung vor.

- eine umfassende Lockerung des Kündigungsschutzes: Fristlose Kündigungen sind zulässig, wenn sich in Krisenzeiten Mitarbeiter betrieblichen Vereinbarungen zur Arbeitszeit- und Lohnreduzierung verweigern. Zudem wird ein neues Massenentlassungsverfahren eingeführt, welches Unterrichtungs- und Konsultationsverfahren vereinfacht und beschleunigt sowie Verjährungsfristen verkürzt.

- Abbau von Bürokratie bei Neueinstellungen, v.a. im Mindestlohnbereich.

- Abschaffung der Gefängnisstrafe für den Tatbestand der Behinderung von Arbeitnehmervertretern.

Das Reformpaket sah zudem tiefgreifende Eingriffe in die erstinstanzlichen Arbeitsgerichte, die sog. Conseil de prud'hommes, sowie eine Höchstgrenze für Abfindungen vor (Serverin, 2016). Diese Reformen wurden jedoch im August 2015 durch den Verfassungsrat als nicht verfassungskonform zurückgewiesen.

Trotz der starken arbeitsmarktregulierenden Eingriffe, die das Loi Macron beinhaltete, gelang es den Gewerkschaften nicht, stärkeren Einfluss auf den Gesetzgebungsprozess zu nehmen oder eine größere Protestbewegung zu mobilisieren. Zwar lehnten die Gewerkschaften den Gesetzesentwurf geschlossen ab, jedoch verfolgten sie jeweils unterschiedliche Strategien. So begrüßte die zweitgrößte französische Gewerkschaft, die sozialdemokratische CFDT, grundsätzlich eine Reform der Arbeitsgerichtbarkeit und die Liberalisierung staatlich beschränkter Berufe, kritisierte jedoch die damit einhergehende Lockerung des Kündigungsschutzes und die Ausweitung der Sonntagsarbeit. Anders als die linken Gewerkschaften (CGT, FO, SUD, FSU) vermieden die reformorientierten Gewerkschaften (CFDT, CFC-CGC, UNSA, CFTC) unter der Führung der CFDT jegliche Konfrontation mit der Regierung und versuchten vielmehr, durch informelle Verhandlungen das Reformpaket abzuschwächen. Durch die traditionelle Verbindung und die starken personellen Überschneidungen zur regierenden PS verfügte die CFDT über einen gewissen informellen Einfluss auf die Reformpolitik der Regierung und somit auch über (prekäre) institutionelle Machtressourcen, welche sie mit einer konfrontativen Strategie nicht gefährden wollte (Syrovatka, 2016). Dagegen lehnten die linken Gewerkschaften unter Führung des CGT das Gesetzespaket in vollem Umfang ab und riefen zu landesweiten Protesten und Streiks auf. 
Bei den Demonstrationen im Dezember 2014 und Januar 2015 gingen landesweit jedoch kaum mehr als 100.000 Menschen gegen die Reformpläne auf die Straße. Die parallel dazu stattfindenden Streiks waren im öffentlichen Leben nicht bemerkbar.

Widerstand gegen die Arbeitsmarktgesetze artikulierte sich auch innerhalb der PS und ihrer Fraktion in der Nationalversammlung. Eine Gruppe von Abgeordneten hatte sich unter der Bezeichnung „Frondeurs“ zu einer innerfraktionellen Opposition gegen die Regierung zusammengefunden. Ihre Kritik am Loi Macron zielte auf die Ausweitung der Sonn- und Nachtarbeitszeitregelungen sowie auf die Lockerung des Arbeitsrechts. Im Parlament kündigten sie an, das Reformpaket abzulehnen. Die Regierung ging nicht auf die Kritik der eigenen Abgeordneten ein, sondern versuchte stattdessen, mit Drohungen und Einschüchterungen die Frondeure zur Zustimmung zu bewegen ${ }^{11}$. Da jedoch die Regierungsmehrheit für das Gesetz nicht mehr gesichert war, entschied sich die Regierung, die Abstimmung über das Reformpaket mit einer Vertrauensfrage zu verknüpfen. Mit Hilfe des §49-3 wurde das Reformprogramm im Februar 2015 ohne Abstimmung und am Parlament vorbei beschlossen.

Die autoritäre Art der Umsetzung machte den geringen Verhandlungskorridor der französischen Regierung für Zugeständnisse an ihre KritikerInnen deutlich. Viel stärker als bei der Rentenreform 2010 verfügte die französische Regierung über einen sehr eingeschränkten Handlungsspielraum für materielle Konzessionen, so dass sie weder die Gewerkschaften noch ihren eigenen linken Parteiflügel in die Reformstrategie einbinden konnte. Dies lag, neben einem zunehmenden innenpolitischen Druck durch Unternehmensverbände ${ }^{12}$ sowie die konservative Opposition, an den Forderungen der EU (Syrovatka, 2016). Der federführende Wirtschaftsminister Emmanuel Macron wollte das Reformpaket daher auch in erster Linie als ein „signal réformiste adressé aux partenaires européens, Allemagne en tête" 13 verstanden wissen, um die europäischen Institutionen und die deutsche Bundesregierung vom eigenen Reformwillen zu überzeugen.

In der Tat hatte die Kommission im Vorfeld der Reformen ein deutliches Signal der Reformbereitschaft von Frankreich gefordert (Sterdyniak, 2015). So kritisierte die Kommission (COM, 2014b) im März 2014 in ihrer eingehenden Überprüfung Frankreichs, im Rahmen des Verfahrens übermäßiger Ungleichgewichte, die geringen Reformfortschritte im Bereich der Arbeitsmarktregulierung:

\footnotetext{
"Further efforts to reduce the cost of labour appear warranted. France is among the countries in the European Union where the cost of labour is the highest. [...] Despite efforts to maintain competitive prices, the competitiveness of French firms is hampered by the high cost of labour. Indeed, French firms' profit margins are the lowest in the EU [...]. Measures planned so far will not be sufficient to fully restore the profitability of non-financial companies" (S. 49)
}

In den LSE der Europäische Kommission (2014a) sowie in den Empfehlungen des Europäischen Rats (2014) im Rahmen des Defizitverfahrens hatten die europäischen Institutionen die Umsetzung zahlreicher Maßnahmen angemahnt. Die Abschaffung der Zugangsbeschränkungen für bestimmte Berufe oder die Lockerung des Kündigungsschutzes waren seit 2013

11 Les Echos, 19.02.2015

12 MEDEF (2014c; 2015a) beklagte noch während des Gesetzgebungsprozesses, dass die vorgesehenen Regelungen deutlich hinter ihren Erwartungen zurückblieben und nicht ausreichten.

13 Emmanuel Macron in Le Monde 16.10.2014 
Teil der europäischen Forderungen (ER, 2013). So findet sich in den LSE die Forderung, dass Frankreich:

„Maßnahmen zur Förderung des Wettbewerbs im Dienstleistungsbereich trifft; ungerechtfertigte Beschränkungen des Zugangs zu und der Ausübung von freiberuflichen Dienstleistungen abschafft, insbesondere was Rechtsform, Beteiligungsstruktur, Quoten und Gebietsbeschränkungen betrifft [...]“ (S. C217/31)

Die Umsetzung des Loi Macron fand zudem vor dem Hintergrund des auslaufenden Defizitverfahrens statt. Auch 2015 konnte Frankreich das Defizitziel nicht einhalten, weshalb v.a. die deutsche Bundesregierung ein klares Reformsignal verlangte. So hatte sie im Vorfeld der Macron-Reformen ihre Zustimmung zu einer Fristverlängerung im Defizitverfahren gegen Frankreich öffentlich an die Durchsetzung tiefgreifender Strukturreformen, v.a. in der Arbeitsmarktregulierung, gekoppelt ${ }^{14}$. Diese offene Drohung an die Adresse der französischen Regierung wurde durch verschiedene institutionelle Initiativen, wie in dem Bericht von Enderlein \& Pisani-Ferry (2014) sowie in zahlreichen medialen Interventionen deutscher Regierungspolitiker, unterstrichen (Lux, 2016). Der starke außenpolitische Druck verengte den Handlungskorridor der Regierungen diskursiv und materiell. Denn wäre es zu einer Abweichung oder Verwässerung des Loi Macron gekommen, hätte dies möglichweise eine geringere Fristverlängerung oder sogar finanzielle Sanktionen zur Folge gehabt, was sich nicht zuletzt auf die wirtschaftliche Situation in Frankreich und damit auf die Refinanzierungsmöglichkeiten auf den internationalen Finanzmärkten ausgewirkt hätte (Lemoine, 2016).

Zugleich erhöhte die öffentliche Debatte über die Wettbewerbsfähigkeit der französischen Wirtschaft den innenpolitischen Handlungsdruck. Unter dem Eindruck schlechter Umfragewerte wollte die Regierung zugleich ihre Handlungs- und Durchsetzungsfähigkeit in der Arbeitsmarktpolitik beweisen. Die Empfehlungen der EU dienten dabei als Impulsgeber sowie als Druck- und Restriktionsinstanz. Vor dem Hintergrund innenpolitischer wie außenpolitischer Spannungen sowie der schwindenden Regierungsmehrheit in der Assemblée nationale setzte die Regierung Valls die Reformen erstmals ohne Konsultierung der Sozialpartner und mit Umgehung des Parlaments durch. Damit stellte das Loi Macron, wie Bazin (2015) betont, sowohl inhaltlich als auch in seiner formellen Umsetzung, einen Paradigmenwechsel in der französischen Reformpolitik dar.

\section{Das Loi El Khomri}

Das zweite große arbeitsmarktregulierende Reformpaket wurde von der französischen Regierung im Februar 2016 vorgestellt und zielte auf eine Reform des Arbeitsrechts und auf eine tiefgreifende Veränderung des Lohnfindungssystems. Vorausgegangen war dem Gesetzentwurf eine breite öffentliche Diskussion über den „Code travail“, der von verschiedenen Seiten als unübersichtlich und kompliziert kritisiert wurde ${ }^{15}$ (Barthélemy \& Cette,

14 FAZ 23.09.2014

15 Dedieu (2016) und Méda (2017) verweisen auf die zentrale Rolle der Think Tanks, TerraNova und Institute Montaigne. Beide hatten in kurzer Zeit zwei einflussreiche Studien veröffentlicht, welche die hohe Arbeitslosigkeit auf das Arbeitsrecht zurückführten. 
2015). Er galt oftmals als Ursache für die hohe Arbeitslosigkeit und selbst linke Arbeitsrechtler und GewerkschafterInnen sprachen sich für seine Vereinfachung und Entschlackung aus (Badinter \& Lyon-Caen, 2015). Der Kritik folgend erarbeitete Jean-Denis Combrexelle (2015) im Auftrag von Manuel Valls im Frühjahr 2015 Vorschläge für eine Reform. Sein Bericht empfahl eine Neuordnung des Tarifvertragssystems und eine Verlagerung sozialpartnerschaftlicher Verhandlungen auf die betriebliche Ebene.

Auf der Grundlage des Berichts erarbeitete das französische Arbeitsministerium einen Gesetzentwurf, welcher tiefgreifende Änderungen im Bereich des Arbeitszeitrechts und eine erneute Stärkung der betrieblichen Ebene bei Vereinbarungen der Sozialpartner vorsah. Die 35-Stunden-Woche blieb offiziell in ihren Grundprinzipien unangetastet, wurde jedoch so stark verwässert, dass ihre Regulierungsfunktion grundlegend in Frage gestellt ist. Konkret zielen die Maßnahmen des Loi El Khomri auf:

- Lockerung und Verwässerung der Überstundenregelung: Auf Grundlage von Betriebsvereinbarungen darf nun - über einen Zeitraum von 16 Wochen - die Wochenarbeitszeit auf 48 Stunden (in Ausnahmefällen sogar auf 60 Stunden) erhöht und tägliche Arbeitszeiten von bis zu 12 Stunden angeordnet werden. Gesetzliche Vergütungsregelungen von Überstunden können durch betriebliche Vereinbarungen abgeschwächt werden. Die 35Stunden-Woche muss nur noch im Durchschnitt von drei Jahren eingehalten werden.

- Stärkung von Vereinbarungen auf der betrieblichen Ebene: Abschaffung des Günstigkeitsprinzips und Umkehrung der Normrangfolge zwischen Tarifvertrag und betrieblichen Vereinbarungen. Betriebliche Vereinbarungen können Branchentarifverträge unterlaufen, auch wenn sie weniger günstige Bedingungen für die ArbeitnehmerInnen bieten. Die Breite von betrieblichen Vereinbarungen wird durch „Entwicklungsvereinbarungen" erweitert. Damit wird die Möglichkeit von offensiven betrieblichen Abkommen geschaffen. „Wirtschaftliche Expansionsbestrebungen“ können über einen Zeitraum von max. zwei Jahren betriebliche Vereinbarungen über Lohnverzicht und höhere Arbeitszeiten begründen.

- Lockerung des Kündigungsschutzes: Die Definition von betriebsbedingten Kündigungen wurde erweitert. Nach Größe des Unternehmens können kleinste wirtschaftliche Verluste Kündigungen begründen.

- Schwächung der Gewerkschaften: Betriebsinterne Referenden über betriebliche Vereinbarungen sind fortan bindend. Betriebsvereinbarungen mit Minderheitsgewerkschaften (Belegschaftsrepräsentanz mind. $50 \%$ ) können damit auch gegen den Widerstand anderer Gewerkschaften abgeschlossen werden.

- Schwächung der Arbeitsgerichte: Abfindungshöhen bei ungerechtfertigter Kündigung werden empfohlen und sollen als nicht verbindliche Orientierung für die Arbeitsgerichte dienen. Jedoch kann es bei Überschreitung der Abfindungsempfehlungen zur richterlichen «Missbrauchskontrolle» kommen.

Die Arbeitsrechtsreform wurde grundlegend von den reformorientierten Gewerkschaften unterstützt. Sie verfolgten eine Strategie der Kooperation und sozialen Partnerschaft, weshalb sie lediglich versuchten durch informelle Verhandlungen mit der Regierung das Reformpaket in einigen Punkten abzuschwächen. Die Strategie war dahingehend erfolgreich, dass etwa die Festsetzung von Obergrenzen für Abfindungen aus dem Gesetzestext entfernt wurde 
(Andolfatto \& Labbé, 2016a). Im Dialog mit den Unternehmensverbänden und der Regierung zielten die reformorientierten Gewerkschaften auf die Ausarbeitung eines gemeinsamen Kompromisses und die Bildung eines wettbewerbskorporatistischen Blocks (Syrovatka 2016, S. 208). Vor allem die CFDT hatte sich schnell auf diese Strategie festgelegt und die Arbeitsrechtsreform in der Öffentlichkeit verteidigt. Anders als die CGT verfolgt die CFDT eine traditionell kooperative Globalstrategie und setzt auf eine Stärkung jener Aushandlungsprozesse, welche den staatlichen Entscheidungen vorgelagert sind (Pernot, 2010; Andolfatto \& Labbé, 2006). Vor diesem Hintergrund erklärt sich die Unterstützung der CFDT für die Arbeitsrechtsreform der Regierung aus dem langfristigen Ziel einer Stärkung des sozialen Dialogs und seiner Institutionalisierung nach dem Vorbild der deutschen Konfliktpartnerschaft. Dahinter steht die strategische Überlegung, durch eine Stärkung der betrieblichen Ebene mehr Handlungsspielraum und Einfluss auf die betriebliche Politik zu erlangen sowie institutionelle Machtressourcen auszubauen (Artus \& Holland, 2013).

Dagegen begleiteten die linken Gewerkschaften den Gesetzgebungsprozess mit Streiks und Protesten, welche den einseitigen angebotspolitischen Charakter des Reformpakets kritisierten und die vollständige Rücknahme des Gesetzes forderten. Sie verfolgten - wie auch schon beim Loi Macron - eine konfrontative Strategie gegenüber der Regierung, welche darauf abzielte, durch Protest auf der Straße und Streiks in den Betrieben, die Regierung zu Zugeständnissen zu zwingen. Daher waren Arbeitsniederlegungen in zahlreichen Branchen ebenso wie Demonstrationen mit mehr als 1,3 Millionen Menschen ${ }^{16}$ Teil der vielfältigen Protestchoreographie gegen das Loi El Khomri. Neben den Gewerkschaften mobilisierten v.a. SchülerInnen und Studierende gegen die Arbeitsrechtsreform und besetzten unter dem Motto „Nuit Debout“ in über 60 Städten monatelang zentrale Plätze. Aufgrund inhaltlicher und habitueller Differenzen blieb eine Zusammenarbeit von linken Gewerkschaften und „Nuit Debout“ jedoch begrenzt (Kokoreff, 2016).

Die Regierung zeigte sich von den Protesten wenig beeindruckt. Premierminister Manuel Valls bezeichnete die Protestierenden als eine „Minderheit, die auf Kosten der Mehrheit das Land blockiert" halte und gegen die man mit Gewalt vorgehen müsse ${ }^{17}$. So reagierte die Regierung auch mit Repression auf die landesweiten Streiks und Proteste. Im Rahmen des, nach den Terroranschlägen in Paris vom November 2015 verhängten, Ausnahmezustands (État d'urgence) kam es zu Hausdurchsuchungen, willkürlichen Verhaftungen, „,präventivem“ Hausarrest gegen AktivistInnen (ohne richterliche Genehmigung) und zu Demonstrationsverboten gegen die Gewerkschaften. Als Folge der Repression ging die Mobilisierung und die Streikbeteiligung zurück.

Ebenso unbeeindruckt zeigte sich die Regierung Valls vom fraktionsinternen Widerstand innerhalb der Nationalversammlung. Aufgrund der offenen Ablehnung der Arbeitsrechtsreform innerhalb der Regierungsfraktion in der Assemblée national wurde schon im Frühjahr 2016 deutlich, dass die Regierung für ihr Gesetzesvorhaben keine Parlamentsmehrheit bekommen würde. Statt jedoch auf die Forderungen der Gewerkschaften und des linken Flügels einzugehen, setzte die Regierung das Reformpaket mithilfe des §49-3 und der Umgehung der Nationalversammlung durch (Audouy, 2016).

Im Vergleich zu den Protesten gegen die Rentenreform von Nicolas Sarkozy 2010 oder gar gegen den Plan Juppé 1995 war die Protestbewegung gegen das Loi El Khomri relativ klein.

17 Le Figaro, 25.05.2016 
Die wiederholt autoritäre Durchsetzung tiefgreifender Reformen in der Arbeitsmarktregulierung macht den eingeschränkten Handlungsspielraum der Regierung deutlich. Zum einen lag dies an einem zugespitzten nationalen Reformdruck in Folge steigender Arbeitslosigkeit und gleichzeitiger Wachstumsschwäche. Waren schon im Wahlkampf 2012 die deutschen Arbeitsmarktreformen der Agenda 2010 als Vorbild für eine umfassende Reform der französischen Wirtschaft propagiert worden, erreichte der Reformdruck im Jahr 2016 seinen Höhepunkt. Vor allem die französischen Kapitalverbände MEDEF und afep hatten sich seit Jahren für eine Reform des Arbeitsrechts sowie eine Aushöhlung der 35-Stunden Woche stark gemacht und übten über öffentliche Interventionen und der Drohung, Arbeitsplätze und Investitionen in die südeuropäischen Krisenstaaten zu verlagern, starken Druck auf die Regierung aus (Les Économistes Atterrés, 2016). So plädierte MEDEF (2015b) schon kurz nach der Verabschiedung des Loi Macrons im Herbst 2015 für eine Reform des Arbeitsrechts und eine Stärkung von Unternehmensvereinbarungen. Der Unternehmensverband begrüßte daher die Reformpläne der Regierung und sicherte seine Unterstützung zu, kritisierte jedoch, dass die Regierung durch die weitere Vergütungsverpflichtung von Überstunden an der 35-StundenWoche festhalte (MEDEF, 2015a). Zugleich befürchtete der Unternehmensverband eine Abschwächung in der angebotsorientierten Stoßrichtung. MEDEF zielte daher während des Gesetzgebungsprozesses auf eine Einflussnahme der öffentlichen Meinung als auch auf die Entscheidungsfindung an sich (Méda, 2017). So verglich der Vorsitzende von MEDEF, Pierre Gattaz, in einem Interview ${ }^{18}$ die CGT mit Terroristen und forderte ein hartes Vorgehen der Polizei gegen die Streikenden. Zugleich drohte MEDEF, ebenso wie der Unternehmensverband der mittelständischen Unternehmen CGPME, mehrfach, die Verhandlungen über die tripartistisch verwaltete Arbeitslosenversicherung zu verlassen ${ }^{19}$.

Zum anderen war die Regierung Valls gezwungen, den Forderungen der EU nachzukommen (Andolfatto \& Labbé, 2016b). In Folge der Terroranschläge von Paris hatte die französische Regierung angekündigt, aufgrund von Mehrausgaben für Polizei und Militär, die Maastrichtkriterien zwangsläufig brechen zu müssen ${ }^{20}$. Die europäischen Institutionen drängten daher zu erneuten Reformen in der Arbeitsmarktregulierung und auch die französische Regierung sah sich genötigt, ein starkes Reformsignal an seine europäischen Partner zu senden (Schreiber \& Dehnert, 2016). Seit 2014 hatten die europäischen Institutionen in ihren LSE eine Flexibilisierung des Lohnbildungssystems, des Kündigungsschutzes sowie eine grundlegende Reform des Arbeitsrechtes gefordert (ER, 2014). Konkret empfahl der Europäische Rat (ER, 2015a):

„dass Frankreich 2015 und 2016 [...] das Arbeitsrecht in einer Weise reformiert, dass stärkere Anreize für Arbeitgeber geschaffen werden; Einstellungen auf der Grundlage unbefristeter Verträge vorzunehmen; Abweichungen von allgemeinen Rechtsvorschriften, insbesondere in Bezug auf die Arbeitszeitregelungen, auf Unternehmens- und Branchenebene erleichtert; [...]“(S. 272).

Auch im Monitoringreport, im Zuge des Defizitverfahrens, wird die französische Regierung dazu aufgefordert, die Maßnahmen des Combrexelles-Reportes vollständig umzusetzen. Die Forderung lautet: ,reduce the costs associated with the implementation of regulations 
concerning working time arrangements, beyond contributing to better align wages with productivity“ (GDECFIN, 2015, S. 33).

Diese „Empfehlungen“ der europäischen Institutionen finden sich in der Reform des Arbeitsrechts wieder. So zielt die Umkehrung der Normrangfolge zwischen Tarifvertrag und betrieblichen Vereinbarungen darauf, die Lohnsetzungsmacht der Gewerkschaften einzuschränken (COM, 2012). Aufgrund des schwachen Organisationsgrads von $8 \%$ und der faktisch kaum vorhandenen betrieblichen Verankerung in privatwirtschaftlichen Unternehmen, werden die Gewerkschaften mit der Durchsetzung des Loi El Khomri stark geschwächt (Andolfatto \& Labbé, 2016b). Die europäischen Institutionen agierten jedoch nicht nur im Vorfeld der Reform als Agenda Setter, sondern übten auch während des Reformprozesses medialen und institutionellen Druck auf die Regierung in Paris aus. So bezeichnete die Europäische Kommission in einem Arbeitspapier vom Februar 2016 die Arbeitsmarktregulierung als Schlüssel, um die Lohnstückkostenentwicklung zu bremsen und die preisliche Wettbewerbsfähigkeit Frankreichs wiederherzustellen. Konkret sei dafür die Durchsetzung der Arbeitsrechtsreform von ,zentraler Bedeutung“ (COM, 2016, S. 2). Zudem äußerten sich EU-Wirtschaftskommissar Pierre Moscovici ${ }^{21}$ und Vizekommissar Valdis Dombrovskis ${ }^{22}$ während des Gesetzgebungsprozesses in Interviews und warnten vor einer Abschwächung des Reformpaketes.

Der Einfluss der europäischen Institutionen bei der Durchsetzung des Loi El Khomri war deutlich größer als noch ein Jahr zuvor bei der Verabschiedung des Loi Macrons. Die harte Linie der französischen Regierung lässt sich damit auch durch die allgegenwärtige Drohung finanzieller Sanktionierung im Rahmen des Europäischen Semesters erklären. Vor allem nach der Ankündigung der französischen Regierung, die Maastrichtkritierien auch 2017 nicht einhalten zu können, stieg der Druck, die geforderten Reformen im Bereich der Arbeitsmarktregulierung auch gegen den Widerstand von Gewerkschaften und Parlament umzusetzen. Zugleich sollte mit dem Gesetz ein Zeichen des Reformwillens gesetzt werden, um die deutsche Bundesregierung zur Zustimmung einer möglichen Verlängerung des Defizitverfahrens und zur Rückkehr einer gemeinsamen deutsch-französischen Europapolitik zu bewegen (Schneider \& Syrovatka, 2017).

Zudem musste die französische Regierung auf die Erwartungshaltungen der globalen Finanzmärkte reagieren. Ein Aufweichen des Reformpakets oder gar finanzielle Sanktionen von EU-Seite hätten den Druck der Finanzmärkte weiter erhöht und eine erneute Herabstufung der Kreditwürdigkeit provoziert. Zuletzt hatte die Agentur Moodys im September 2015 die Kreditwürdigkeit des Landes herabgesetzt und den Schritt mit der hohen Arbeitslosigkeit und den geringen Reformanstrengungen in der Arbeitsmarktregulierung begründet ${ }^{23}$.

Verstärkt wurde der ,äußere“ Reformdruck durch die innenpolitische Einflussnahme der nationalen Unternehmensverbände (Méda, 2017; Andolfatto \& Labbé, 2016a). Vor allem MEDEF begleitete sowohl über informelle Kanäle als auch medial den Gesetzgebungsprozess und versuchte, eine Abschwächung oder Verwässerung zu verhindern. Viele Forderungen der europäischen Institutionen, wie eine Vereinfachung des Arbeitsrechts und die Schaffung von Abweichmöglichkeiten bei der Arbeitszeitregelung, korrespondierten

2120 minute, 18.05.2016

22 Euractiv, 02.04.2016

23 Les Echos, 21.09.2015, S.4 
mit langjährigen Forderungen der Unternehmensverbände. Bereits 2007 hatte MEDEF in seinem Positionspapier „Besoin d'air" die Aufwertung von Verhandlungen auf der Unternehmensebene sowie die faktische Abschaffung der 35-Stunden-Woche gefordert (Parisot, 2007). Positionen, die 2014 in der Reformagenda „France 2020“ erneut formuliert wurden (MEDEF, 2014b). Zusammen mit den reformorientierten Gewerkschaften und dem Mehrheitsflügel der regierenden PS hatte sich eine innenpolitische „Interessenkoalition“ (Méda, 2017, S. 40) formiert, welche über ein weitverzweigtes und einflussreiches Netzwerk verfügte und die Umsetzung der Arbeitsrechtsreform grundlegend unterstützte. Als Träger des Reformprojektes verstärkte der innenpolitische „wettbewerbskorporatistische Block“ (Syrovatka, 2016, S. 209) die Forderungen der europäischen Institutionen und ermöglichte die Umsetzung der Arbeitsrechtsreform in seiner spezifischen Form.

\section{Fazit}

Das Beispiel der französischen Arbeitsmarktregulierung unter François Hollande zeigt, dass sich durch die Etablierung eines krisenkonstitutionalistischen Arrangements auf der europäischen Ebene ein arbeitsmarktpolitischer Interventionismus herausgebildet hat, welcher, je nach spezifischer Form, direkten oder indirekten Einfluss auf die nationale Arbeitsmarktregulierung ausübt. Das Beispiel zeigt zudem, dass selbst die schwächste Form des arbeitsmarktpolitischen Interventionismus im Rahmen der LSE des Europäischen Semesters starke Einschränkungen des nationalen Handlungsspielraums in der Arbeitsmarktregulierung mit sich bringt. Sowohl Reichweite als auch Qualität des arbeitsmarktpolitischen Interventionismus hängen dabei stark von den Trägern der Reformvorschläge innerhalb des Landes sowie vom nationalen Kräfteverhältnis $a b$. So gab es nicht nur von den nationalen Unternehmensverbänden eine breite Unterstützung für die Reformen, sondern auch von Teilen der Gewerkschaften. Beide Reformen wären nicht in der radikalen Form durchsetzbar gewesen, hätten die linken (CGT u.a.) und die reformorientierten Gewerkschaften (CFDT u.a.) gemeinsam agiert. Dies war jedoch nicht der Fall, der Regierungskurs wurde von den reformorientierten Gewerkschaften weitestgehend unterstützt. Dazu kommt der starke Einfluss der nationalen Kapitalverbände auf den öffentlichen Diskurs, aber auch auf die Europäische Kommission. So legen die offensichtlichen Ähnlichkeiten zwischen den Forderungen der nationalen Kapitalverbände und dem LSE der europäischen Institutionen nahe, dass sich vor allem MEDEF nicht nur in die Politikformulierung auf der nationalen Ebene, sondern ebenso auf europäischer Ebene einschreiben konnte (vgl. Woll, 2011).

Das Beispiel macht zudem deutlich, dass der arbeitsmarktpolitische Interventionismus zu einer massiven Schwächung der Gewerkschaften und ihrer Einflussmacht auf die nationale Arbeitsmarkt- und Lohnpolitik führt. Er fördert und forciert in unterschiedlicher Qualität eine Dezentralisierung des Tarifvertragssystems und eine Verbetrieblichung der Lohnfindung. Damit wird die Position der Gewerkschaften untergraben und ein Arbeitsmarkt geschaffen, in dem kollektive Arbeitsbeziehungen eine untergeordnete Rolle spielen. Dagegen wurden marktgetriebene Mechanismen in den nationalen Arbeitsmarktregulierungen gefördert (Keune, 2015). Dies zeigte sich primär im Loi El Khomri. Diese Reform des Arbeitsrechts wird mittelfristig zu einer Erosion der Branchentarifverträge und zu einem Einbruch 
der jetzt schon stagnierenden Lohnentwicklung führen. Durch den geringen Organisierungsgrad und der kaum vorhandenen Verankerung in privatwirtschaftlichen Betrieben wird auch die Abschaffung des Günstigkeitsprinzips bei gleichzeitiger Umkehrung der Normrangfolge zwischen Tarifvertrag und betrieblichen Vereinbarungen zu einer weiteren Schwächung der Organisations- und institutionellen Macht der französischen Gewerkschaften führen (Andolfatto \& Labbé, 2016b).

Die angebotspolitische und gewerkschaftsfeindliche Stoßrichtung des arbeitsmarktpolitischen Interventionismus ist in der gesamten EU zu beobachten (Schulten \& Müller 2013). Während im Rahmen des Europäischen Semesters dieser Prozess allmählich und in mehreren kleineren Schritten durchgesetzt wird, wurden in jenen Ländern, welche unter der Beobachtung der Troika stehen, die Tarif- und Lohnfindungssysteme radikal verändert oder gar vollständig zerstört (Cruces, Trillo \& Leonardi, 2015). Damit nimmt die EU, über den arbeitsmarktpolitischen Interventionismus in seinen verschiedenen Ausprägungen, Einfluss auf Bereiche, in die sie sich bisher nicht eingemischt hatte und in denen sie auch offiziell keine Zuständigkeiten auf Grundlage der EU-Verträge besitzt (Rödl, 2012). Damit hat sich nicht nur der Charakter europäischer Arbeitsmarkt- und Beschäftigungspolitik fundamental verändert, sondern auch Reichweite und Verbindlichkeit wurden deutlich ausgebaut.

Zudem ist es mit der Etablierung arbeitsmarktregulierender Interventionsinstrumente zu einer weiteren Autoritarisierung des Staates gekommen. Dies wird am hier bearbeiteten französischen Beispiel deutlich, da die arbeitsmarktregulierenden Reformen nur durch die Umgehung des Parlaments und fundamentaler demokratischer Prinzipien durchgesetzt werden konnten. Die Verhärtung der politischen Form führt jedoch zu einer Delegitimierung demokratischer Institutionen und mittelfristig zu einer Krise des gesamten politischen Systems.

Derzeit wird auf europäischer Ebene, als Folge der zunehmenden Desintegrationstendenzen, über weitere Schritte in der europäischen Krisenbearbeitung beraten. Sowohl der 5Präsidentenbericht als auch das Weißbuch der Europäischen Kommission zur Zukunft der EU schlagen Maßnahmen zur Ausweitung und Intensivierung des arbeitsmarktpolitischen Interventionismus vor. Ein Beispiel dafür sind bereits verabschiedete Wettbewerbsräte, welche den Einfluss des arbeitsmarktpolitischen Interventionismus auf die nationalen Lohnpolitiken erheblich ausweiten werden. Die Überlegungen verdeutlichen, dass der krisenkonstitutionalistische Umbau der EU noch nicht abgeschlossen ist und sich möglichweise in einer neuen Stufe angebotspolitischer Radikalisierung befindet (Schneider \& Syrovatka, 2017).

\section{Literatur}

Aghion, P., Cette, G. \& Cohen, É. A. (2014). Changer de modèle. Paris: Odile Jacob.

Andolfatto, D. \& Labbé, D. (2006). La transformation des syndicats français. Vers un nouveau « modèle social »? Revue française de science politique, 56, 281-297. doi: 10.3917/rfsp.562.0281

Andolfatto, D. \& Labbé, D. (2016a). La réforme, la rue et les syndicats. Commentaire, 156, 825-832. doi: $10.3917 /$ comm.156.0825

Andolfatto, D. \& Labbé, D. (2016b). Un printemps social français. Le Débat, 191, 67-75. doi: $10.3917 /$ deba.191.0067

Artus, I. \& Holland, J. (2013). Von der Belebung des toten Ritters in seiner Rüstung: Coalition building und Gewerkschaftsproteste in Frankreich. In S. Schmalz \& L. Dörre (Hrsg.), Comeback der Gewerkschaften? Machtressourcen, innovative Praktiken, internationale Perspektiven (S. 131147). Frankfurt am Main: Campus Verlag. 
Audouy, L. (2016). La révision de l'article 49 alinéa 3 de la Constitution à l'aune de la pratique. Revue française de droit constitutionnel, 107 (3), e1-e22. doi: 10.3917/rfdc.107.0745

Badinter, R. \& Lyon-Caen, A. (2015). Le travail et la loi. Paris: Fayard.

Barthélemy, J. \& Cette, G. (2015). Réformer le droit du travail. Paris: Odile Jacob.

Bazin, F. (2015). La loi « Macron» un coup d'essai. Aprés-demain, 34 (2), 6-7.

Bieling, H.-J. (2011). Vom Krisenmanagement zur neuen Konsolidierungsagenda der EU. PROKLA. Zeitschrift für kritische Sozialwissenschaft, 163, 173-194.

Bieling, H.-J. \& Brand, U. (2015). Competitiveness or emancipation? Rethinking regulation and (counter-)hegemony in times of capitalist crisis. In R. Westra, D. Badeen \& R. Albritton (Hrsg.), The future of capitalism after the financial crisis: The varieties of capitalism debate in the age of austerity (S. 184-204). London: Routledge.

Bieling, H.-J. \& Hüttmann, M. G. (2016). Zur Einführung: Staatlichkeit der Europäischen Union in Zeiten der Finanzkrise. In H.-J. Bieling \& M. Große Hüttmann (Hrsg.), Europäische Staatlichkeit: Zwischen Krise und Integration (S. 11-30). Wiesbaden: Springer VS. doi: 10.1007/978-3-658-03790-1_1

Brand, U., Görg, C. \& Wissen, M. (2007). Verdichtungen zweiter Ordnung. Die Internationalisierung des Staates aus einer neo-poulantzianischen Perspektive. PROKLA. Zeitschrift für kritische Sozialwissenschaften, 147, 217-234.

Clift, B. \& Ryner, M. (2014). Joined at the hip, but pulling apart? Franco-German relations, the Eurozone crisis and the politics of austerity. French Politics, 12 (2), 136-163. doi: org/10.1057/fp.2014.8

Combrexelle, J.-D. (2015). La négociation collective, le travail et l'emploi (Rapport au Premier minister). Abgerufen von France Stratégie:

http://www.strategie.gouv.fr/sites/strategie.gouv.fr/files/atoms/files/fs_rapport_missionjdc_0809 2015.pdf

Cruces, J. Álvarez, I., Trillo, F. \& Leonardi, S. (2015). Impact on the euro crisis on wages and collective bargaining in southern Europe - a comparison of Otaly, Portugal and Spain. In G. van Gyes $\&$ T. Schulten (Hrsg.), Wage bargaining under the new European economic governance: Alternative strategies for inclusive growth (S. 93-138). Brüssel: etui.

Dedieu, F. (2016). Gouvernement de coalition. Une fausse solution. Le Débat, 188 (1), 96-104. doi: 10.3917/deba.188.0096

Degryse, C., Jepsen, M. \& Pochet, P. (2013). The Euro crisis and its impact on national and European social policies. Brüssel: etui.

Duval, G. (2013). Made in Germany. Le modèle allemand au-delà des mythes. Paris: SEUIL.

Dyson, K. (2010). Norman's Lament. The Greek and Euro Area Crisis in Historical Perspective. New Political Economy, 15, 597-608. doi: 10.1080/13563467.2010.492853

Enderlein, H. \& Pisani-Ferry, J. (2014). Reformen, Investitionen und Wachstum: Eine Agenda für Frankreich, Deutschland und Europa. Abgerufen von:

http://www.bmwi.de/Redaktion/DE/Publikationen/Studien/empfehlung-enderlein-pisaniferry.html

Erne, R. (2015). European Economic Governance. Auf dem Weg zu einer erzwungenen Integration nationaler Arbeitsbeziehungen? In S. Pernicka (Hrsg.), Horizontale Europäisierung im Feld der Arbeitsbeziehungen (S. 183-200). Wiesbaden: Springer VS. doi: 10.1007/978-3-658-07556-9_7

Europäische Kommission (COM) (2012). Labour Market Developments in Europe. Luxemburg: European Union.

Europäische Kommission (COM) (2014a). Empfehlung für eine Empfehlung des Rates zum nationalen Reformprogramm Frankreichs 2014 mit einer Stellungnahme des Rates zum Stabilitätsprogramm Frankreichs 2014. Brüssel: European Commission. 
Europäische Kommission (COM) (2014b). Macroeconomic imbalances. France 2014. Brüssel: European Commission.

Europäische Kommission (COM) (2015). Recommendation for a Council Recommendation with a view to bringing an end to the excessive government deficit in France. Brüssel: European Commission.

Europäische Kommission (COM) (2016). Commission Staff Working Document - Country Report France 2016. Including an In-Depth Review on the prevention and correction of macroeconomic imbalances. Brüssel: European Commission.

Europäischer Rat (ER) (2013). Empfehlung des Rates vom 9. Juli 2013 zum nationalen Reformprogramm Frankreichs 2013 mit einer Stellungnahme des Rates zum Stabilitätsprogramm Frankreichs für die Jahre 2012 bis 2017. Brüssel: European Council.

Europäischer Rat (ER) (2014). Council Recommendation of 8 July 2014 on the National Reform Programme 2014 of France and delivering a Council opinion on the Stability Programme of France, 2014. Brüssel: European Council.

Europäischer Rat (ER) (2015a). Council Recommendation of 14 July 2015 on the 2015 National Reform Programme of France and delivering a Council opinion on the 2015 Stability Programme of France. Brüssel: European Council.

Europäischer Rat (ER) (2015b). Council Recommendation with a view to bringing an end to the excessive government deficit in France. Brüssel: European Council.

Europäischer Rat (ER) (2016). Empfehlung des Rates vom 12. Juli 2016 zum nationalen Reformprogramm Frankreichs 2016 mit einer Stellungnahme des Rates zum Stabilitätspro-gramm Frankreichs 2016. Brüssel: European Council.

GDECFIN (2015). France - Review of progress on policy measures relevant for the correction of macroeconomic imbalances. Brüssel: European Commission.

Heine, F. \& Sablowski, T. (2015). Zerfällt die Europäische Währungsunion? Handels- und Kapitalverflechtungen, Krisenursachen und Entwicklungsperspektiven der Eurozone. PROKLA. Zeitschrift für kritische Sozialwissenschaften, 181, 563-592.

Heinrich, M. (2015). EU governance in crisis. A cultural political economy perspective on European crisis management 2007-2014. Comparative European Politics, 13, 682-706. doi: org/10.1057/cep.2014.49

Heinrich, M. \& Jessop, B. (2015). Interpretations of the EU crisis and their policy implementation. In B. Jessop, B. Young \& C. Scherrer (Hrsg.), Financial cultures and crisis dynamics (S. 278-293). London: Routledge.

Jabko, N. \& Massoc, E. (2012). French capitalism under stress. How Nicolas Sarkozy rescued the banks. Review of International Political Economy, 19, 562-585. doi: 10.1080/09692290.2011.638896

Keune, M. (2015). The effects of the EU's assault on collective bargaining. Less governance capacity and more inequality. Transfer: European Review of Labour and Research, 21, 477-483. doi: $10.1177 / 1024258915603571$

Kokoreff, M. (2016). Nuit debout sur place. Les Temps Modernes, 691 (5), 157-176. doi: $10.3917 / \mathrm{ltm} .691 .0157$

Lemoine, B. (2016). L'ordre de la dette. Les infortunes de l'État et la prospérité du marché. Paris: La Découverte.

Les Économistes Atterrés (2016). La loi El Khomri ou comment en finir avec le Code du travail. Abgerufen von:

http://www.atterres.org/article/la-loi-el-khomri-ou-comment-en-finir-avec-le-code-du-travail

Lux, J. (2017). Disciplining large member states during the crisis. Analyzing the discursive strategies of the EU and German actors on France. Critical Policy Studies, 2 (1), 1-17.

doi: $10.1080 / 19460171.2016 .1208107$ 
Méda, D. (2017). Pourquoi la gauche de gouvernement a-t-elle oublié le travail? Mouvements, 89 (1), 32-42. doi: 10.3917/mouv.089.0032

MEDEF (2014a). 1 million d'emploi... c'est possible. Abgerufen von: http://www.1milliondemplois.fr/uploads/Livre-jaune-1-milion-emplois-c-est-possible_MEDEF2014.pdf

MEDEF (2014b). France 2020. Manifest pour faire gagner la France. Abgerufen von: http://www.medef-somme.fr/files/2014/09/France_2020_MEDEF-52pages_edition1.pdf

MEDEF (2014c). Projet de loi pour la croissance et l'activité des pistes pertinentes, mais des précisions à apporter. Communiqué de presse. Abgerufen von: http://www.medef-somme.fr/projet-deloi-pour-la-croissance-et-lactivite-des-pistes-pertinentes-mais-des-precisions-a-apporter-2.html

MEDEF (2015a). Loi pour la croissance et l'activité réformer est un exercice difficile, mais la réforme est indispensable. Abgerufen von:

http://www.medef.com/fr/communique-de-presse/article/loi-pour-la-croissance-et-l-activitereformer-est-un-exercice-difficile-mais-la-reforme-est-indispensable

MEDEF (2015b). Pierre Gattaz sur France 2 « Le code du travail est trop compliqué ». Communiqué du presse. Abgerufen von:

http://www.medef-somme.fr/pierre-gattaz-sur-france2-le-code-du-travail-est-trop-complique.html

Müller, T. \& Platzer, H.-W. (2016). Gewerkschaften und Arbeitsbeziehungen im Europa der Krise. Strukturdaten und Entwicklungstrends im Vergleich. In F. Bsirske, K. Busch, O. Höbel, R. Knerler \& D. Scholz (Hrsg.), Gewerkschaften in der Eurokrise: Nationaler Anpassungsdruck und europäische Strategien (S. 10-44). Hamburg: VSA.

Müller, T. \& Schulten, T. (2015). European economic governance and its intervention in national wage development and collective bargaining. In S. Lehndorff (Hrsg.), Divisive integration: The triumph of failed ideas in Europe - revisited (S. 331-363) Brüssel: etui.

Müller, T., Schulten, T. \& Zuckerstätter, S. (2015). Wages and economic performance in Europe. In G. van Gyes \& T. Schulten (Hrsg.), Wage bargaining under the new European economic governance: Alternative strategies for inclusive growth (S. 259-282). Brussels: etui.

Neumann, A. (2017). La bureaucratie liquide. Variations, 20, 1-25. doi: 10.4000/variations. 810

Oberndorfer, L. (2014). A New Economic Governance through Secondary Legislation? Analysis and Constitutional Assessment. In N. Bruun, K. Lörcher \& I. Schömann (Hrsg.), The Economic and Financial Crisis and Collective Labour Law in Europe (S. 25-54). Oxford: Hart Publishing.

Overbeek, H. (2012). Sovereign Debt Crisis in Euroland. Root Causes and Implications for European Integration. The International Spectator, 47, 30-48. doi: 10.1080/03932729.2012.655006

Parisot, L. (2007). Besoin d'air. Paris: Le Seuil.

Pernot, J.-M. (2010). Syndicats. Lendemains de crise? Paris: Folio.

Poulantzas, N. (2002). Staatstheorie. Politischer Überbau, Ideologie, autoritärer Etatismus. Hamburg: VSA.

Rödl, F. (2012). Kompetenzverstoß der Europäischen Union. Die Sanktionierung der Eurostaaten im Rahmen der Excessive Imbalance Procedure. Zeitschrift für Staats- und Europawissenschaften, 10, 99-109. doi: 10.5771/1610-7780-2012-1-99

Rödl, F. \& Callsen, R. (2015). Kollektive soziale Rechte unter dem Druck der Währungsunion. Schutz durch Art. 28 EU-Grundrechtecharta? Frankfurt am Main: Bund-Verlag.

Schmidt, V. A. (2013). Speaking to the Markets or to the People? A Discursive Institutionalist Analysis of the EU's Sovereign Debt Crisis. The British Journal of Politics and International Relations, 16, 188-209. doi: 10.1111/1467-856X.12023

Schneider, E. \& Syrovatka, F. (2017). Der Fünf-Präsidenten-Bericht - die »Vollendung « des europäischen Desintegrationsprozesses? Leviathan, 45, 55-80. doi: 10.5771/0340-0425-2017-1-55 
Schreiber, B. \& Dehnert, S. (2016). Die Reform des Arbeitsrechts spaltet die Linke Frankreichs. Abgerufen von:

https://www.igmetall.de/20160419_FES_Frankreich_Info_Die_Reform_des_Arbeitsrechts_spalt et_die_Linke_Fra_a947af1b95096c0357154d83f1316c13df6f6514.pdf

Schulten, T. \& Müller, T. (2013). Ein neuer europäischer Interventionismus? Die Auswirkungen des neuen Systems der europäischen Economic Governance auf Löhne und Tarifpolitik. Wirtschaft und Gesellschaft, 39, 291-321.

Serverin, E. (2016). Décryptage la réforme de la justice prud'homale, d'une critique à l'autre. Le Droit Ouvrir, 812, 118-127.

Simonazzi, A., Ginzburg, A. \& Nocella, G. (2013). Economic relations between Germany and southern Europe. Cambridge Journal of Economics, 37 (3), 653-675. doi: 10.1093/cje/bet010

Sterdyniak, H. (2015). À propos de la loi Macron, Les notes, 49, 1-10. Abgerufen von: https://www.ofce.sciences-po.fr/pdf/notes/2015/note49.pdf

Syrovatka, F. (2016). Die Reformpolitik Frankreichs in der Krise. Arbeitsmarkt- und Rentenpolitik vor dem Hintergrund europäischer Krisenbearbeitung. Wiesbaden: Springer VS. doi: 10.1007/978-3-658-14060-1

van Gyes, G. \& Schulten, T. (2015). Introduction. In G. van Gyes \& T. Schulten (Hrsg), Wage bargaining under the new European economic governance. Alternative strategies for inclusive growth (S. 9-22). Brüssel: etui.

Woll, C. (2011). Le lobbying à rebours. L'influence du politique sur la stratégie des grandes entreprises. Paris: Presses de Sciences Po. 


\section{DuEPublico}

Duisburg-Essen Publications online
UNIVERSITÄT

DE S S SEN R G

offen im Denken

Ub $\mid \begin{aligned} & \text { universitäts } \\ & \text { bibliothek }\end{aligned}$

Dieser Text wird über DuEPublico, dem Dokumenten- und Publikationsserver der Universität Duisburg-Essen, zur Verfügung gestellt. Die hier veröffentlichte Version der EPublikation kann von einer eventuell ebenfalls veröffentlichten Verlagsversion abweichen.

DOI: $\quad$ 10.3224/indbez.v25i1.04

URN: urn:nbn:de:hbz:464-20200702-151248-4

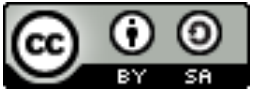

Dieses Werk kann unter einer Creative Commons Namensnennung - Weitergabe unter gleichen Bedingungen 4.0 Lizenz (CC BY-SA 4.0) genutzt werden. 